\title{
Promising Therapies for Fungal Infection Based on the Study to Elucidate Mechanisms to Cope with Stress in Candida Species
}

\author{
Kazuyuki Hirai ${ }^{1}$, Tatsuya Inukai ${ }^{2,3}$ and Hironobu Nakayama ${ }^{1}$ \\ ${ }^{1}$ Faculty of Pharmaceutical Sciences, Suzuka University of Medical Science \\ ${ }^{2}$ Department of Chemotherapy and Mycoses, National Institute of Infectious Diseases \\ ${ }^{3}$ Diseases Division of Pathology, Immunology and Microbiology, Graduate School of Medicine, The University of Tokyo
}

\begin{abstract}
In recent years, the incidence of fungal infections has been increasing, particularly among patients with immune systems compromised by human immunodeficiency virus infection, organ transplantation, and/or chemotherapy for cancer. Current therapies for treating systemic fungal infection have limited effectiveness and have created problems of adverse reactions and drug resistance. These issues therefore motivate us to develop novel antifungals. Elucidation of stress response mechanisms and virulence factors in pathogenic fungi is required in developing an effective antifungal strategy. There are actually numerous studies concerning various stress responses in several important fungal pathogens. Among these responses, we focused on stress response for iron starvation to identify potential targets for novel antifungals because iron starvation occurs in blood, where pathogenic fungi often infect. Here we show recent progress of studies on iron homeostasis in Candida species, especially focusing on Candida glabrata, and propose novel antifungal targets.
\end{abstract}

Key words : Candida, iron metabolism, iron starvation, molecular targets, stress response

\section{Introduction}

Many deep-seated fungal infections can occur as opportunistic infections in immunocompromised patients, but have been steadily increasing due to clinical situation including increase of ageing population and advanced medical treatments. The number of cases where patients have died after contracting these infections is also not insignificant. In general, Candida infection is one of the major cases of deep mycoses, Candida species. is located above the causative agents isolated from patients attacked by septicemia. Invasive aspergillosis, which is caused by the Aspergillus genus, has a high mortality rate in advanced immunocompromised patients". Cryptococcosis, which is caused by the Cryptococcus genus, can also occur in healthy individuals, and can sometimes develop into severe meningitis in immunocompromised patients ${ }^{2}$. The number of treatments for fungal infections that are in current use is limited for various reasons including the difficulty of setting a target molecule for eukaryotes (unlike bacteria and the like), and the poor understanding of their infection mechanisms. Furthermore, the use of these therapeutic agents can itself sometimes cause problems including serious side-effects and narrowing of the antifungal spectrum, so the development of therapeutic agents for fungal infections is urgently needed.

Criteria for the selection of a target molecule for an antifungal agent are importance for growth, less homology with humans, and contribution to pathogenicity. In particular, information on the essentiality of fungal growth is the most important among the selection criteria, but since it is necessary to determine the importance in the host body environment, simple in vitro culture environments do not provide sufficient information. This should be kept in mind before reaching any conclusions ${ }^{3}$. Considering the complexity of 
Table 1

\begin{tabular}{ll}
\hline Stress from host & Fungi response, etc. \\
\hline $\begin{array}{l}\text { High temperature } \\
\text { Oxidative stress } \\
\text { Low pH }\end{array}$ & Resistance mechanisms \\
Protease & \\
Restricted sources of nutrients & Hyphal formation \\
& $\begin{array}{l}\text { Maintenance of homeostasis (including uptake from external sources) } \\
\text { Degradation of tissue by hydrolytic enzymes: protease secretion }\end{array}$ \\
Drug resistance & Overexpression/mutation of target enzymes \\
& Expression of drug efflux pumps, etc. \\
\hline
\end{tabular}

host body environments, it is possible that fungi are subjected to various environmental stresses, including high temperatures, immune attack, and restricted sources of nutrients including ions (Table 1). Therefore, to develop effective treatments, numerous studies are using a variety of fungi to elucidate their response mechanisms under environmental stress ${ }^{4}$. Of the many different stress response mechanisms, the responses to restricted sources of nutrients have a strong association with disseminated infection. In this review, we therefore focused on the stress response mechanism to cope with iron starvation. After showing recent progress of studies in Candida species (especially C. glabarata) including our results, we propose novel antifungal targets.

\section{Characteristics of C. glabrata}

C. glabrata is an opportunistic fungal pathogen that causes severe invasive infection, and is the second most frequently species isolated after $C$. albicans. Isolated ratio is $10 \%$ for invasive Candida and over $20 \%$ for blood infection, and patients infected with $C$. glabrata are categorized as poor prognosis ${ }^{5}$. Also, C. glabrata is not only a clinically important strain, but it also has highly reliable genomic information ${ }^{6,7)}$, resulting in easy genetic manipulation and analysis. Furthermore, infection models including mice and silkworms have been established. With this background, C. glabarata can be recognized as one of the most important model organisms for study of pathogenic fungi. Therefore, the studies to clarify the association between stress response mechanisms and infec- tion mechanisms are in progress, and the reports of various studies on stress responses have already been published. The topics of these studies include the response mechanism to oxidative stress to evade attack from immunocompetent cells ${ }^{8}$, calcineurin signaling pathways associated with various stress responses ${ }^{9}$, and stress response mechanisms of the endoplasmic reticulum ${ }^{10}$.

\section{Response to iron starvation in fungi (mainte- nance of iron homeostasis)}

Since iron is used in various reactions including redox reactions in cells, an excess or shortage of iron affects multiple systems including not only the cell metabolism but also the functioning of mitochondria and the integrity of the cell wall. Therefore, maintaining homeostasis of iron concentration is important for the survival of pathogenic fungi as well as other organisms. In particular, the maintenance of iron homeostasis in the stress response to iron starvation is strongly associated with pathogenesis and is therefore receiving a lot of attention ${ }^{11}$. Although $C$. glabrata is a fungus that infects in the gastrointestinal tract, it can spread from there to the blood vessels in immunocompromised patients (e. g., organ transplant recipients), resulting in disseminated hematogenous infections. Although free iron concentration in the gastrointestinal tract is sufficient for growth, but the concentration in the blood is kept much lower due the action of ironbinding proteins like transferrin and ferritin. Therefore, a response to iron starvation stress is one of the environmental responses it needs to cause disseminated infection. The maintenance of 


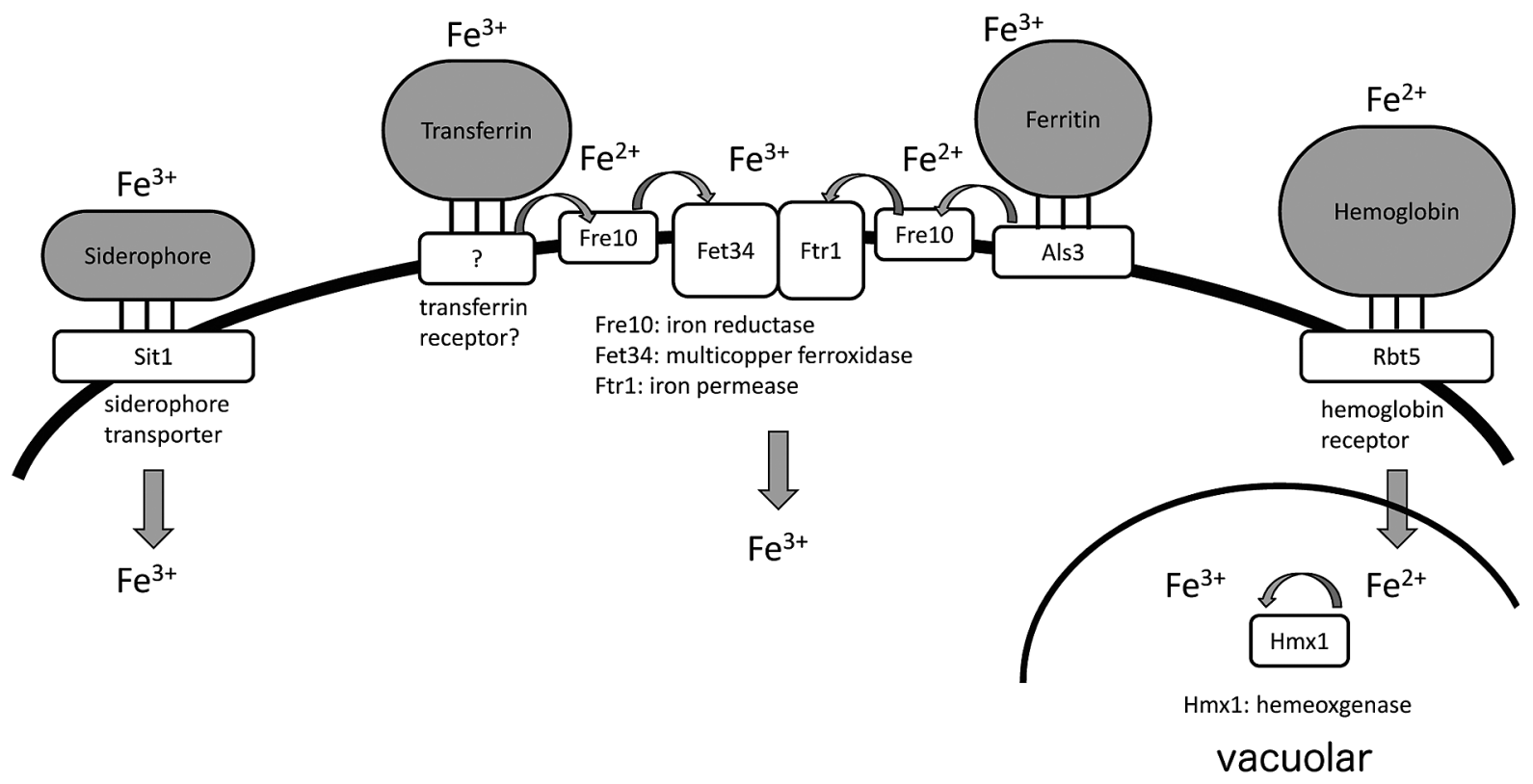

Fig. 1 Iron acquisition mechanism in C. albicans.

iron homeostasis is easier to understand if it is separated into the maintenance of iron uptake and the maintenance of intracellular iron concentration. The following sections present separate discussions of recent trends in the elucidation of molecular maintenance mechanisms, including novel antifungal targets.

\section{Iron uptake in the Candida genus}

Fungus acquires iron via siderophore transporters (siderophore is a small peptide molecule generated by some bacteria and fungi). Since fungus has hemolytic activity similar that of bacteria, free iron generated by lysis of blood cells was taken by siderophores. It has also been reported the lines of evidence that show the direct iron removal from the host iron binding protein via ferritin, transferrin receptors and hemoglobin receptors ${ }^{12}$. It has been assumed that similar uptake mechanism is conserved in Candida species. Therefore, studies to identify the iron uptake mechanism in C. albicans are actively carried out ${ }^{13)}$ (Fig. 1). However, the factors participating in iron removal from host iron binding proteins have not yet been identified in $C$. glabrata, and only the siderophore transporter the SIT1 gene has been isolated ${ }^{14)}$. Also, the ornithine-N5-oxygenase orthologue that plays an essential role in the siderophore biosynthesis pathway has also not been identified ${ }^{15}$. Like $C$. albicans and budding yeast Saccharomyces cerevisiae, C. glabrata utilizes the siderophores of other fungi (hydroxamate type siderophore) when it takes up iron via Sit1 ${ }^{14)}$. It has been shown that the sit1-deleted strain (sit1 $\Delta$ ) shows has a lower survival rate within macrophages, suggesting that inhibitors of siderophore transporter would be promising antifungal drugs. However, considering C. glabrata utilizes siderophore of other fungi, we can not conclude that $C$. glabrata always utilizes a siderophore transporters as a method to acquire iron. Furthermore, other pathogenic fungi including $C$. albicans have multiple iron uptake mechanisms, and study using mouse infection model revealed that siderophore-deleted strain (sit1 $\Delta$ /sit1 $\Delta$ ) was not avirulent. On the basis of these results, it is considered that it is not optimal to target only siderophore transporters ${ }^{16)}$.

\section{Mechanisms to maintain iron concentration in fungal cells}

The regulation of iron concentration in fungal cells consists iron uptake/release control, storage in organelles, and changes in metabolism. The studies using $S$. cerevisiae are carried out most actively, showing the most advanced evidence. Therefore, we show here a brief overview of recent studies on iron uptake/release mechanisms in S. cerevisiae.

It has been demonstrated that iron uptake is activated and repressed by transcription factors Aft1/Aft2 and Yap5 respectively, resulting in the expression of gene clusters in the corresponding 

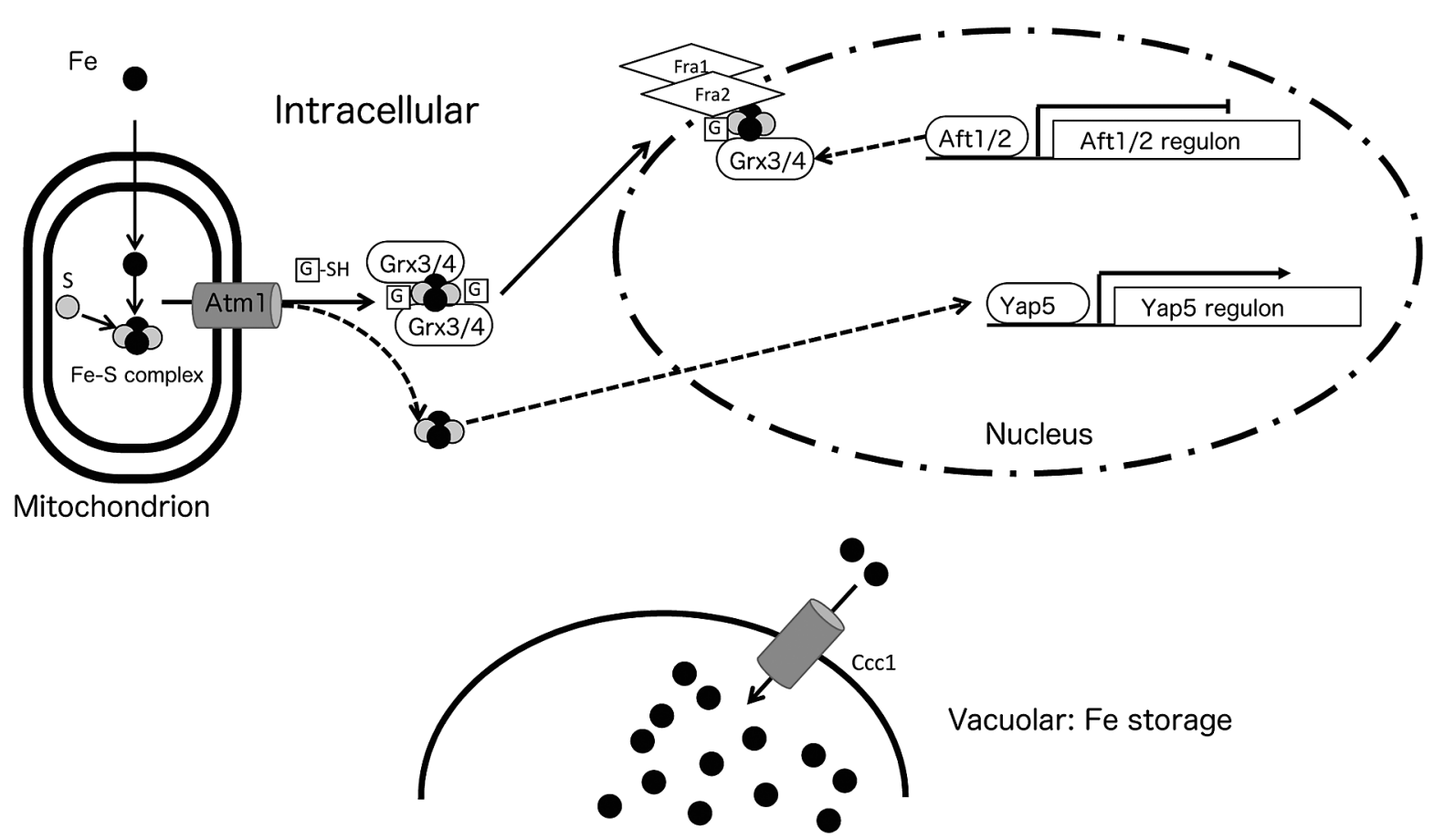

Fig. 2 Regulation of iron concentration in yeast.

regulons ${ }^{17)}$. When iron starvation occurs, it is known that Aft1/Aft2 is transported into the nucleus by karyopherin Pse1, which belongs to a family of nuclear transport receptors ${ }^{18}$. On the other hand, when there is a high iron concentration in the cell, it has been reported that iron is stored in vacuoles and other organelles via the transporter $\mathrm{Ccc1}$, with Fe-S clusters produced by mitochondria acting as signaling molecules ${ }^{19)}$. These Fe-S clusters are released from the mitochondria via Atm1, sent to the nucleus after binding with glutathione (GSH) and monothiol glutaredoxin $\mathrm{Gr} \times 3 / \mathrm{Gr} \times 4$, and bind with Aft1/Aft2 to remove Aft1/Aft2 from the DNA and stop the transcription of gene clusters in the regulon (Fig. 2). It has also been shown that post-transcriptional regulation via mRNA binding protein Cth1/Cth2 works when iron starvation occurs ${ }^{20)}$. The CTH1 and CTH2 gene are located in the Aft1/Aft2 regulon, and their transcripts, $\mathrm{Cth} 1$ and $\mathrm{Cth} 2$, bind mRNAs harboring AU rich mRNA sequence (ARE: $A U$-rich element) and their binding complexes are digested. The mRNAs harboring ARE encode the proteins related to iron utilization processes that include respiration, the TCA cycle, heme synthesis, amino acid synthesis, sterol and fatty acid synthesis, and the formation of Fe-S clusters by mitochondria. Therefore, Cth1 and Cth2 stop the syntheses of these proteins at the posttranscriptional level, resulting in supression of iron utilization. Also, since the mRNAs of the CTH1 and $\mathrm{CTH} 2$ gene harbor ARE, they also regulate their own transcription, thereby preventing excessive suppression of iron utilization.

Further research is awaited to determine whether or not the above mechanism is conserved in most pathogenic fungi including Candida, but according to predictions based on genomic sequences of $C$. glabrata, they have transcription factors similar to $S$. cerevisiae and may participate in metabolic regulation of intracellular iron. Also, as in prokaryotes, since the gene clusters associated with iron metabolism have a regulon structure and are controlled at the transcription level, it is thought that the mechanism is not the same as the post-transcriptional regulation by iron regulatory protein (IRP) in mammals (Fig. 3). From the above consideration, it seems highly likely that inhibitors of Aft1/Aft2 orthologues could be used for the therapy for fungal infections.

\section{Adaptation by metabolic changes due to iron starvation}

As described in the former section, iron is utilized in cellular processes including respiration, the TCA cycle, heme synthesis, amino acid synthesis, sterol and fatty acid synthesis, and mitochondrial Fe-S cluster synthesis. Therefore, reducing the concentration of iron in a cell 


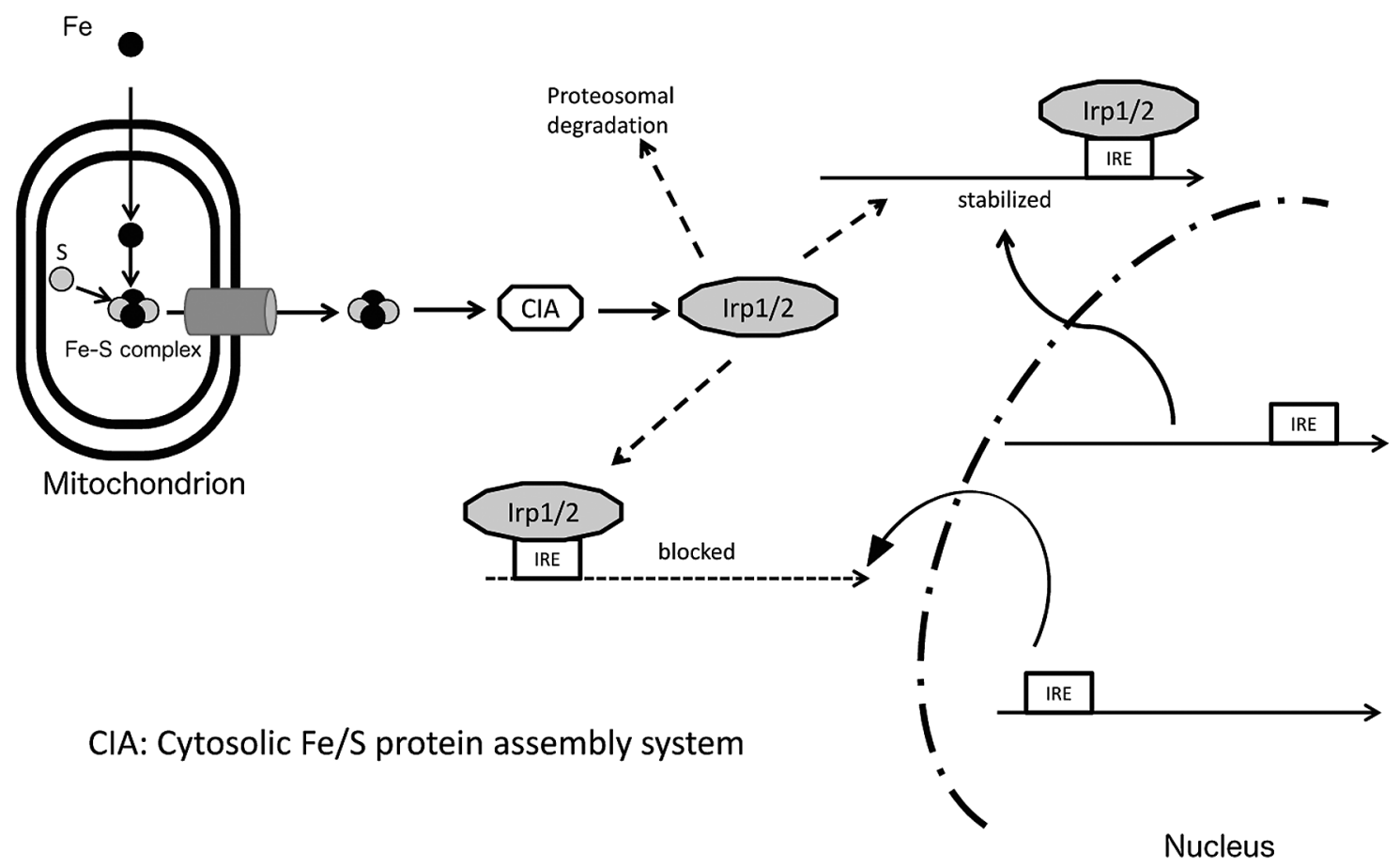

Fig. 3 Regulation of iron concentration in mammals.

hinders many biological reactions, causing major changes in metabolism. From a transcriptome analysis of $C$. glabrata using a DNA microarray, we focused on the increased expression of ATG32 in response to iron starvatoin, and showed that the degradation of mitochondria (mitophagy) is mediated by $\operatorname{Atg} 32^{211}$. Further study is needed to confirm whether this mitophagy happens because of disfunction of mitochondria due to iron starvation or due to the supply of iron, but since an atg32-deleted strains (atg32 $\Delta$ ) has lower pathogenicity, it is thought that the inhibition of mitophagy is also one of the targets for antifungal therapy. We also discovered that during iron starvation there are changes in the expression of genes associated with iron metabolism and sterol uptake (Table 2). In fact, iron starvation causes reduce of heme iron, inactivation of the enzyme Erg11 (sterol 14 alpha demethylase) participating in ergosterol biosynthesis, resulting in ergosterol depletion. As result of this, the cells take in exogenous sterol to rescue sterol depletion (Fig. 4) ${ }^{22,23}$. Since serum component such as transferrin functions as iron chelators, this metabolic change is also observed by the addition of serum, and sterol uptake during iron starvation is thought to be related to disseminated infections of $C$. glabrata. It has been confirmed that an ABC transporterdeleted strain, aus $1 \Delta$ (which plays a central role in the uptake of exogenous sterol), has reduced pathogenicity ${ }^{23}$, and sterol uptake during iron starvation has not been so far reported in other Candida species ${ }^{24}$. It is unlikely that sterol uptake is a promising target for antifungal therapy. On the other hand, it is suggested that transcription factor Upc2 (which is activated in response to iron starvation to regulate sterol homeostasis) and its orthologues are associated with pathogenicity and drug resistance even in $C$. albicans, Cryptococcus neoformans and AspergilIus fumigatus, so we can expect that inhibiting this transcriptional activity could be a target of antifungal therapy. In fact, it has been reported that screening of inhibitors of Upc2 function using C. albicans has been in progress ${ }^{25,26)}$.

\section{Conclusion}

In this paper, we showed the stress response to iron starvation, and from the molecular mechanism, as the target molecule of antifungal therapies, we considered transcription factors, proton pump and transporter involved in iron metabolism. Anti-fungal drugs inhibiting function(s) of transcription factors have not developed so far. Therefore, such inhibitors are expected to be novel type of antifungal agents although some issues including their delivery to nucleus or 


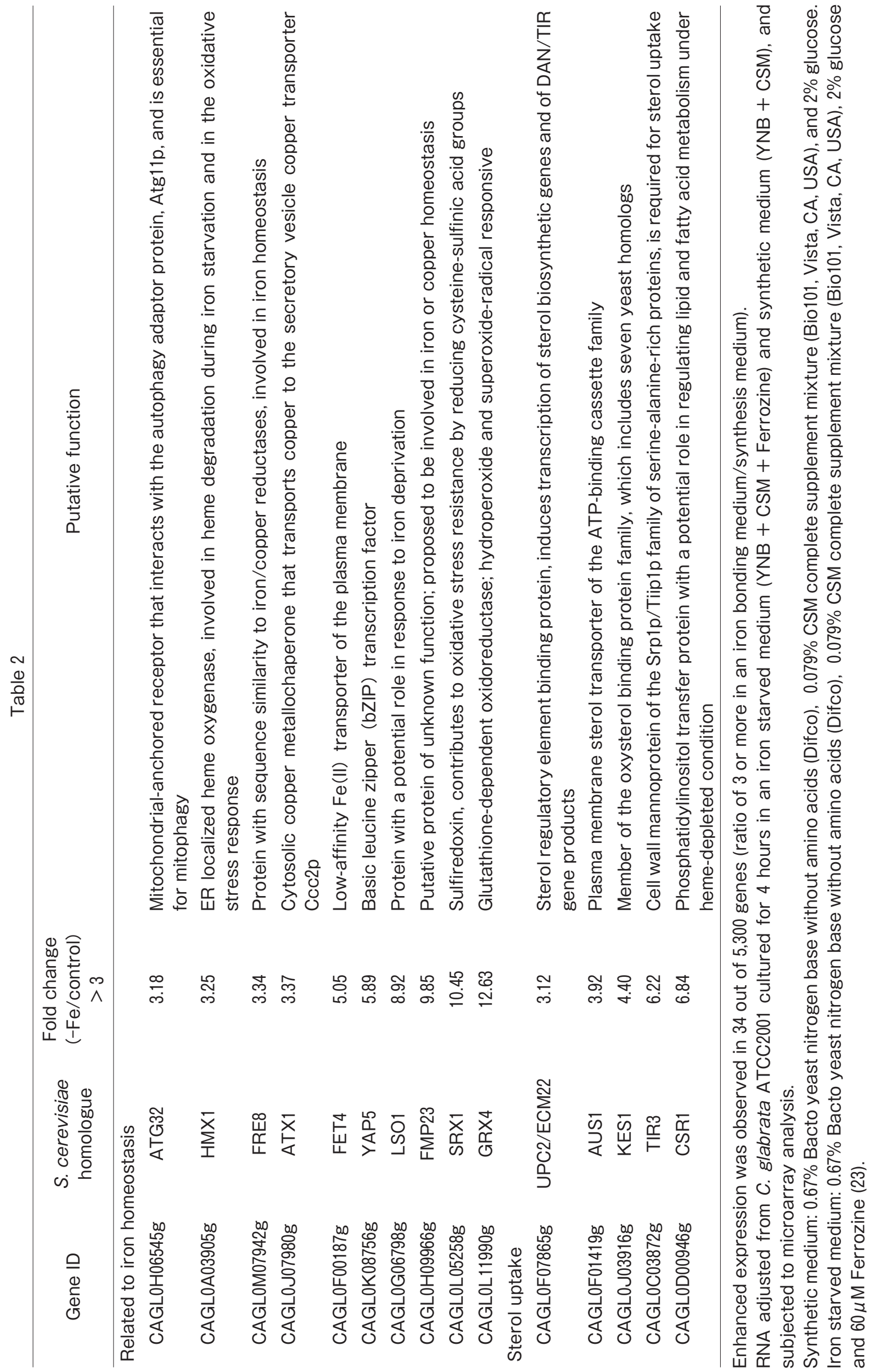




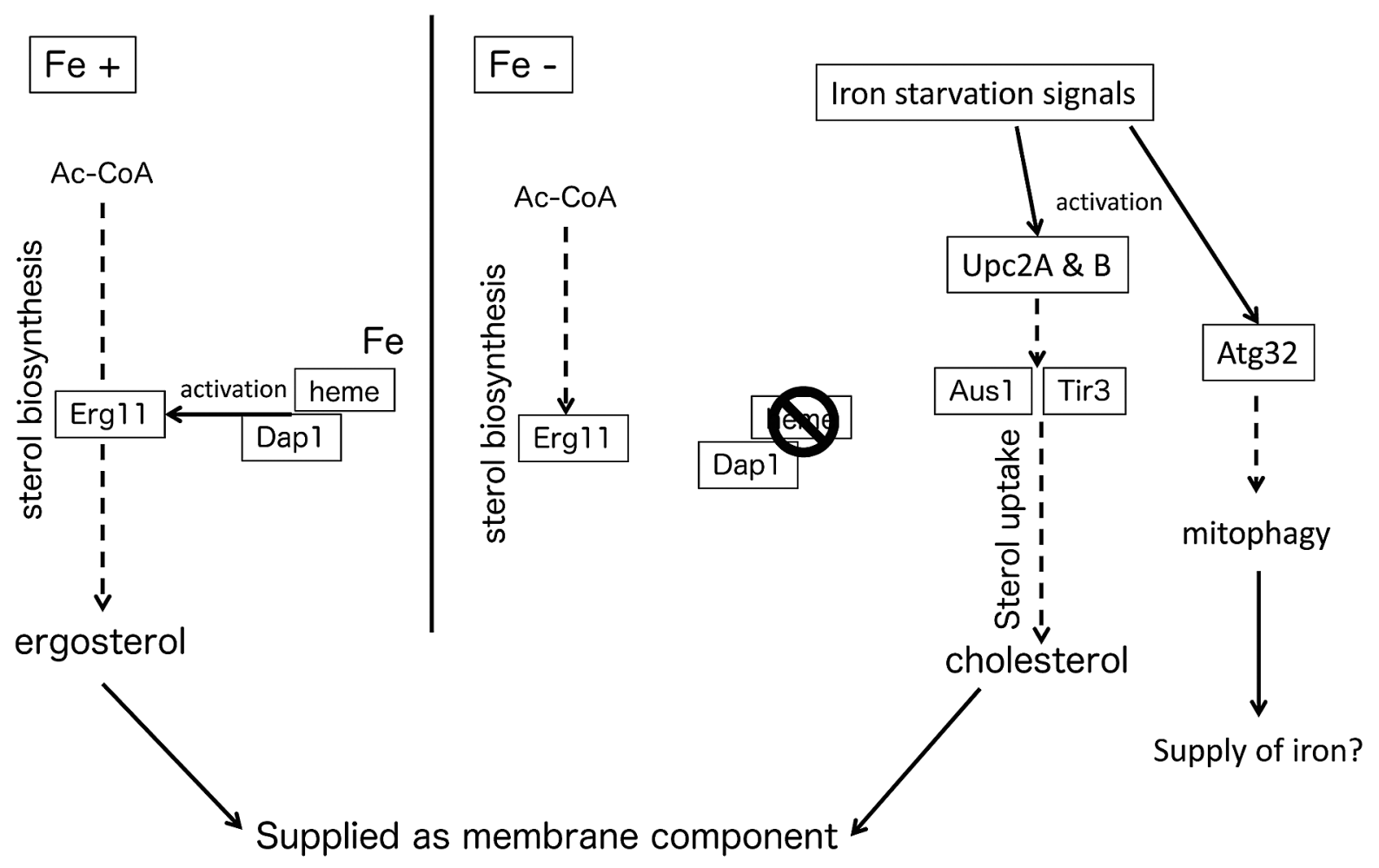

Fig. 4 Sterol homeostasis during iron starvation.

cytosol aroud nuclear should be overcome. In addition, the factors participating in various cellular responses have been conserved between fungi and mammalians since fungi are eukaryotes. In fact, drugs such as calcineurin inhibitors (cyclosporine or FK506) and HSP90 inhibitors (geldanamycin) have also been reported to have a certain level of antifungal activity ${ }^{27}$. Based on these findings, It would be alternative approach to hunt antifungal activity against the compounds that are developed or have been developed as possible inhibitors of iron starvation response.

Furthermore, in clinical practice, infections are often accompanied by bacterial infections. Responses to iron starvation are also exhibited by bacteria, and in some areas the research into bacteria is more advanced than the research into fungi, including the lines of evidence to show iron acquisition using host factors including catecholamines such as epinephrine or norepinephrine ${ }^{28,29}$. To identify mechanism of fungal iron acquisition using host factor(s) might provide us to uncover conserved mechanism (s) of iron acquisition in "unicellular organisms" including fungi and bacteria. Then, such studies could provide us a chance to develop better therapy for fungal infections. Therefore, it is worth keeping an eye on develop- ments in the research of bacteria.

\section{Conflict of interest}

None.

\section{References}

1) Marr KA, Carter RA, Crippa F, Wald A, Corey L: Epidemiology and outcome of mould infections in hematopoietic stem cell transplant recipients. Clin Infect Dis 34: 909-917, 2002.

2) Chayakulkeeree M, Perfect JR: Cryotococcosis. Infect Dis Clin North Am 20: 507-544, 2006.

3) Nakayama H, Izuta M, Nakayama N, Arisawa M, Aoki $Y$ : Depletion of the squalene synthase (ERG9) gene does not impair growth of Candida glabrata in mice. Antimicrob Agents Chemother 44: 2411- 2418, 2000.

4) O'Meara TR, Cowen LE: Hsp90-dependent regulatory circuitry controlling temperature-dependent fungal development and virulence. Cell Microbiol 16: 473-481, 2014.

5) Pfaller MA, Diekema DJ: Epidemiology of invasive candidiasis: a persistent public health problem. Clin Microbiol Rev 20: 133-163, 2007.

6) Aoyama T, Nakayama H, Ueno K, Inukai T, Tanabe K, Nagi M, Bard M, Chibana H: Genome-wide survey of transcriptional initiation in the pathogenic fungus, Candida glabrata. Genes Cells 19: 
478-503, 2014

7) Linde J, Duggan S, Weber M, Horn F, Sieber $P$ Hellwig D, Riege K, Marz M, Martin R, Guthke R, Kurzai O: Defining the transcriptomic landscape of Candida glabrata by RNA-Seq. Nucleic Acids Res 43: 1392-1406, 2015.

8) Roetzer A, Klopf E, Gratz N Marcet-Houben M, Hiller E, Rupp S, Gabaldón T, Kovarik P, Scgüller C: Regulation of Candida glabrata oxidative stress resistance is adapted to host environment. FEBS lett 585: 319-327, 2011.

9) Miyazaki T, Izumikawa K, Nagayoshi $Y$, Saijo T, Yamauchi S, Morinaga Y, Seki M, Kakeya H, Yamamoto Y, Yanagihara K, Miyazaki Y, Kohno S: Functional characterization of the regulators of calcineurin in Candida glabrata. FEMS Yeast Res 11: 621-630, 2011.

10) Miyazaki $T$, Nakayama $H$, Nagayoshi $Y$, Kakeya $H$, Kohno S: Dissection of Ire1 functions reveals stress response mechanisms uniquely evolved in Candida glabrata. PLoS Pathog 9: e1003160, 2013.

11) Ding C, Festa RS, Sun TS, Wang ZY: Iron and copper as virulence modulators in human fungal pathogens. Mol Microbiol 93: 10-23, 2014.

12) Potrykus J, Ballou ER, Childers DS, Brown AJ: Conflicting interests in the pathogen-host tug of war: fungal micronutrient scavenging versus mammalian nutritional immunity. PLoS Pathog 10: e1003910, 2014.

13) Noble SM: Candida albicans specializations for iron homeostasis: from commensalism to virulence. Curr Opin Microbiol 16: 708-715, 2013.

14) Nevitt $T$, Thiele $D J$ : Host iron withholding demands siderophore utilization for Candida glabrata to survive macrophage killing. PLoS Pathog 7: e1001322, 2011.

15) Haas H: Molecular genetics of fungal siderophore biosynthesis and uptake: the role of siderophores in iron uptake and storage. Appl Microbial Biotechnol 62: 316-330, 2003.

16) Heymann $P$, Gerads $M$, Schaller M, Dromer $F$, Winkelmann G, Ernst JF: The siderophore iron transporter of Candida albicans (Sit1p/Arn1p) mediates uptake of ferrichrome-type siderophores and is required for epithelial invasion. Infect Immun 70: 5246-5255, 2002.

17) Outten CE, Albetel AN: Iron sensing and regulation in Saccharomyces cerevisiae: Ironing out the mechanistic details. Curr Opin Microbiol 16: 662668, 2013.

18) Ueta R, Fukunaka A, Yamaguchi-Iwai Y: Pse1p mediates the nuclear import of the ironresponsive transcription factor Aft1p in Saccharomyces cerevisiae. J Biol Chem 278: 50120-50127, 2003.

19) Lill R, Hoffmann B, Molik S, Pierik AJ, Rietzschel N, Stehling O, Uzarska MA, Webert H, Wilbrecht C,
Mühlenhoff U: The role of mitochondria in cellular iron-sulfur protein biogenesis and iron metabolism. Biochim Biophys Acta 1823: 1491-1508, 2012.

20) Philpott CC, Leidgens S, Frey AG: Metabolic remodeling in iron-deficient fungi. Biochim Biophys Acta 1823: 1509-1520, 2012.

21) Nagi M, Tanabe K, Nakayama H, Ueno K, Yamagoe S, Umeyama T, Ohno H, Miyazaki Y: Irondepletion promotes mitophagy to maintain mitochondrial integrity in pathogenic yeast Candida glabrata. Autophagy 12: 1259-1271, 2016.

22) Hosogaya N, Miyazaki T, Nagi M, Tanabe $K$, Minematsu A, Nagayoshi Y, Yamauchi S, Nakamura S, Imamura Y, Izumikawa K, Kakeya H, Yanagihara K, Miyazaki Y, Kugiyama K, Kohno S: The heme-binding protein Dap1 links iron homeostasis to azole resistance via the P450 protein Erg11 in Candida glabrata. FEMS Yeast Res 13: 411-421, 2013.

23) Nagi M, Tanabe K, Ueno K, Nakayama H, Aoyama $\mathrm{T}$, Chibana H, Yamagoe S, Umeyama T, Oura T, Ohno H, Kajiwara S, Miyazaki Y.: The Candida glabrata sterol scavenging mechanism, mediated by the ATP-binding cassette transporter Aus1p, is regulated by iron limitation. Mol Microbiol 88: 371-381, 2013.

24) Zavrel M, Hoot SJ, White TC: Comparison of sterol import under aerobic and anaerobic conditions in three fungal species, Candida albicans, Candida glabrata, and Saccharomyces cerevisiae. Eukaryot Cell 12: 725-738, 2013.

25) Bien CM, Espenshade PJ: Sterol regulatory element binding proteins in fungi: hypoxic transcription factors linked to pathogenesis. Eukaryot Cell 9: 352-359, 2010.

26) Gallo-Ebert C, Donigan M, Stroke IL, Swanson RN, Manners MT, Francisco J, Toner G, Gallagher D, Huang CY, Gygax SE, Webb M, Nickels JT Jr: Novel antifungal drug discovery based on targeting pathways regulating the fungus-conserved Upc2 transcription factor. Antimicrob. Agents Chemother 58: 258-266, 2014.

27) Zhang J, Liu W, Tan J, Sun Y, Wan Z, Li R: Antifungal activity of geldanamycin alone or in combination with fluconazole against Candida species. Mycopathologia 175: 273-279, 2013.

28) Freestone PP, Lyte M, Neal CP, Maggs AF, Haigh $\mathrm{RD}$, Williams $\mathrm{PH}$ : The mammalian neuroendocrine hormone norepinephrine supplies iron for bacterial growth in the presence of transferrin or lactoferrin. J Bacteriol 182: 6091-6098, 2000.

29) O'Donnell PM, Aviles H, Lyte M, Sonnenfeld G: Enhancement of in vitro growth of pathogenic bacteria by norepinephrine: importance of inoculum density and role of transferrin. Appl Environ Microbiol 72: 5097-5099, 2006. 